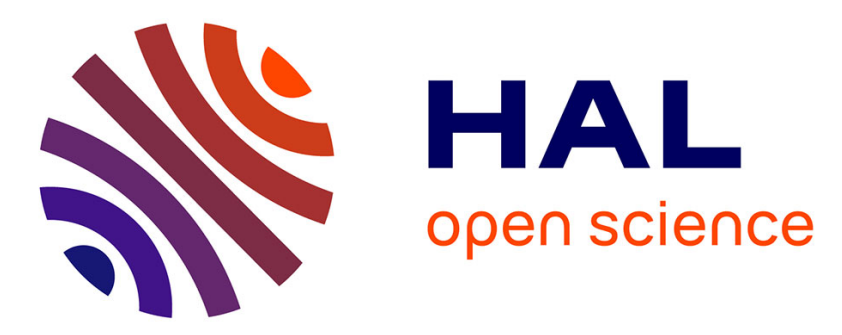

\title{
La conception atemporaliste de la création dans les débats de philosophie analytique contemporaine Paul Clavier
}

\section{To cite this version:}

Paul Clavier. La conception atemporaliste de la création dans les débats de philosophie analytique contemporaine. Revue des sciences philosophiques et theologiques, 2018, 102 (1), pp.37. 10.3917/rspt.1021.0037 . hal-02976751

\section{HAL Id: hal-02976751 \\ https://hal.univ-lorraine.fr/hal-02976751}

Submitted on 30 Oct 2020

HAL is a multi-disciplinary open access archive for the deposit and dissemination of scientific research documents, whether they are published or not. The documents may come from teaching and research institutions in France or abroad, or from public or private research centers.
L'archive ouverte pluridisciplinaire HAL, est destinée au dépôt et à la diffusion de documents scientifiques de niveau recherche, publiés ou non, émanant des établissements d'enseignement et de recherche français ou étrangers, des laboratoires publics ou privés. 
Paul Clavier : La conception atemporaliste de la création dans les débats de philosophie analytique contemporaine

Résumé: Longtemps éclipsée, la question de la compatibilité de la (pre)science divine avec la liberté a été exhumée dans les débats de philosophie analytique. Les promoteurs de la logique temporelle ont proposé un argument d'incompatibilité qui continue d'exercer la sagacité des commentateurs. L'objet de cet article est de suggérer que la conception atemporaliste de Dieu, dont on explique pourquoi elle est si peu en vogue aujourd'hui, est à même, sinon de résoudre complètement, au moins d'atténuer considérablement ces problèmes de compatibilité, qui rejoignent le problème de l'articulation de la souveraineté absolue de Dieu avec la contribution authentique des causes secondes.

Mots clés :

Prescience, liberté, création continuée, philosophie analytique de la religion, éternité, atemporalité, occasionnalisme, molinisme, causes secondes

La philosophie contemporaine de la religion a exhumé, entre autres, deux questions que l'histoire de la philosophie académique avait enterrées : la question de la compatibilité de la (pre)science divine avec la libertél; et celle de la compatibilité de la souveraineté absolue de Dieu avec la contribution authentique des causes secondes ${ }^{2}$. Ces deux questions n'en

\footnotetext{
${ }^{1}$ Voir par exemple Freedom, Fatalism and Foreknowledge, ed. by John Martin Fischer and Patrick Tood, Oxford University Press, 2015.

${ }^{2}$ Voir par exemple Hugh J. McCann, selon qui la part active dans toute opération des créatures n'entraîne pas l'occasionnalisme (Creation and the Sovereignty of God, Bloomington, Indiana: Indiana University Press, 2012, pp. 30-35). McCann souligne que si la causalité seconde était productrice d'existence, alors Dieu perdrait le contrôle sur l'opération des créatures (operari sequitur esse) Les causes secondes, dans l'ordre naturel, ne confèrent pas l'existence, mais modifient des réalités déjà existantes.
} 
font d'ailleurs peut-être qu'une. Si l'on considère que la souveraineté absolue de Dieu implique son omniscience, et que certaines créatures ont un pouvoir d'agir librement comme cause seconde, le problème de la conciliation entre prescience et la liberté n'est qu'un cas particulier du problème de la conciliation entre souveraineté divine et causes secondes. La doctrine de la création continuée et ses implications se rappelle alors au bon souvenir du philosophe contemporain: si d'instant en instant, l'ensemble des réalités créées est reconduit dans l'existence par une opération du créateur, reste-t-il quelque chose à l'autonomie des causes secondes?

L'objet de cet article est d'évoquer la résurgence de ces difficultés dans la philosophie analytique contemporaine de la religion, et de suggérer que la conception atemporaliste de Dieu, peu en vogue dans cette même philosophie, est à même, sinon de résoudre complètement, au moins d'atténuer considérablement ces difficultés.

\section{Une question éclipsée}

La question de la compatibilité de la (pre)science divine avec la liberté des créatures aura agité maints esprits, d'Augustin à Molina. Elle semble avoir connu dans la suite une éclipse. Il entre peu à peu dans les esprits qu'on a affaire à une énigme insoluble. C'est en tous cas l'idée popularisée par Milton, qui campe au livre second les occupations des esprits infernaux tandis que Satan s'en va visiter la terre :

D'autres siégeaient à part, retirés sur un mont, Absorbés qu'ils étaient en des pensées sublimes Examinant dans leurs profonds raisonnements : Providence, Prescience, Volonté et Destin, Destin fixé, volonté libre, science absolue, Perdus, dedans ces labyrinthes sans issue ${ }^{3}$

\footnotetext{
3 "Others apart sat on a hill retired, In thoughts more elevate, and reasoned high Of Providence, Foreknowledge, Will, and FateFixed fate, free will, foreknowledge absolute, And found no end, in wandering mazes lost » (Paradise Lost, Liv.II).
} 
La responsabilité de Descartes dans le contournement, voire l'ajournement définitif sur ces questions n'est pas mince. Descartes revendique l'incompréhensibilité de l'accord de notre libre arbitre avec la préordination divine. Descartes recommande de ne plus s'embarrasser de ces difficultés ${ }^{4}$. Il argue de la disproportion entre notre pensée (finie) et la toute-puissance de Dieu, «par laquelle il a non seulement connu de toute éternité ce qui est ou qui peut être, mais il l'a aussi voulu». Nous avons beau «connaître clairement et distinctement que cette puissance est en Dieu », nous ne saurions " comprendre tellement son étendue que nous puissions savoir comment elle laisse les actions des hommes entièrement libres et indéterminées ». Bref, si nous sommes assurés du fait, non seulement nous ignorons comment, mais nous ne pouvons que l'ignorer. Pour autant, Descartes refuse également la solution qui consisterait à nier le libre-arbitre, ou à affirmer que le libre-arbitre est compatible avec la prédétermination des actions. A l'assurance de la préordination de toutes les actions par le vouloir divin, répond l'assurance « de la liberté et de l'indifférence qui est en nous », assurance telle que « la toute-puissance de Dieu ne nous doit point empêcher de la croire $»^{5}$. Deux assurances et une ignorance de principe sur leur compatibilité : le sujet était clos pour longtemps. Bossuet se fera lui aussi le chantre de l'incompréhensible conciliation du libre-arbitre et de la Providence prédéterminante et presciente. «La première règle de notre logique, c'est qu'il ne faut jamais abandonner les vérités une fois connues, quelque difficulté qui survienne quand on veut les concilier; mais qu'il faut au contraire, pour ainsi parler, tenir fortement comme les deux bouts de la

\footnotetext{
${ }^{4}$ « nous pourrions aisément nous embarrasser en des difficultés très grandes si nous entreprenions d'accorder la liberté de notre volonté avec ses ordonnances, et si nous tâchions de comprendre, c'est-à-dire d'embrasser et comme limiter avec notre entendement, toute l'étendue de notre libre arbitre et l'ordre de la Providence éternelle. » (Principes de la philosophie I, art.40, AT IX-2, p. 42)

${ }^{5}$ « Car nous aurions tort de douter de ce que nous apercevons intérieurement et que nous savons par expérience être en nous, parce que nous ne comprenons pas une autre chose que nous savons être incompréhensible de sa nature. » (Principes de la philosophie I, art. 41, AT IX-2, p. 42).
} 
chaîne, quoiqu'on ne voit pas toujours le milieu par où l'enchaînement se continue $»^{6}$.

Les philosophes analytiques du XXème siècle, exhumant ces questions théologiques, ne se contenteront pas de cette conciliation sous X.

\section{L'argument d'incompatiblité (de la prescience divine avec la liberté)}

Le premier problème posé par la prescience divine, est celui de son incompatibilité avec le libre arbitre des créatures. Supposons que Dieu ait la science infaillible des futurs contingents libres. La version temporaliste de cette omniscience divine a de fâcheuses conséquences.

Définissons tout d'abord ce qu'on entend par infaillibilité :

$\mathrm{D}$ est infaillible $={ }_{\text {Def }} \mathrm{si} \mathrm{D}$ sait que $\mathrm{p}$, alors inévitablement $\mathrm{p}$

On peut alors formuler un argument d'incompatibilité de la prescience divine avec la liberté. C'est l'argument standard qu'on retrouve depuis Arthur Prior, fondateur de la logique temporelle (tense logic) ${ }^{7}$ :

$\mathrm{Si}$, à $t_{0}$, antérieur à $t$, Dieu a la science infaillible, de ce qu'un agent $\mathrm{A}$ accomplira à $t$, alors il est inévitable que A accomplisse cette action à $t$ S'il est inévitable que A accomplisse telle action à t, alors A n'aurait pas pu agir autrement à $t$

A accomplit librement une action à $t$ seulement s'il est vrai que A aurait pu agir autrement

Si Dieu a la science infaillible à $t_{0}$, antérieur à $t$, de ce que A accomplit à $t$, alors A n'accomplit pas librement son action à $t$.

\section{La parade proposée par Guillaume d'Ockham consistait à considérer les}

\footnotetext{
${ }^{6}$ Traité du Libre-arbitre, ch. IV, Cuvres Complètes de Bossuet, Paris, Méquignon Junior et J. Leroux, Gaume Frères, 1846, tome V, p. 122. Tout ce chapitre semble développer l'article 41 des Principes de Descartes. "Quand il nous aurait caché le moyen dont il se sert pour conduire notre liberté, s'ensuivrait-il qu'on dût pour cela ou nier qu'il la conduise, ou dire qu'il la détruise en la conduisant ?»(p. 120).

7 Prior, A. N. : Time and modality, Oxford, Oxford University Press 1957, et Past, Present and Future, Oxford, Oxford University Press, 1967. Prior conclut que Dieu n'a pas la prescience d'actes libres. Un Dieu temporel ne peut que pronostiquer des actes libres, pas les connaître. Rien ne peut être dit futurum (allant se produire) avant d'être présent dans ses causes au point de ne plus pouvoir être empêché (nothing can be said to be truly 'going to happen' (futurum) until it is so 'present in its causes'as to be beyond stopping) « The Formalities of Omniscience » dans Arthur Prior, Papers on Time and Tense, London, Oxford University Press, 1968,p. 52. Prior conteste que « Pour tout $p$, si (c'est le cas que) $p$, Dieu a[it] toujours su que ce serait le cas que $p »($ p. 43).
} 
connaissances passées de Dieu comme des faits dépendants des actes futurs de $\mathrm{A}^{8}$. Les propositions décrivant la science de Dieu peuvent donc être «vocalement» au passé (on énonce en effet que Dieu savait ou a su que...) mais ils sont « réellement» au futur. Ce que Dieu sait à $t_{0}$, antérieur à $t$, de ce que $\mathrm{A}$ accomplit ultérieurement, à $t$, en réalité Dieu, à $t$, l'aura su à $t_{0} \ldots$

Les philosophes analytiques contemporains, friands de concepts originaux, ont semble-t-il donné une seconde chance à cette solution ockhamienne, en formulant la distinction entre faits souples et faits durs (d'abord Nelson Pike, puis Marilyn McCord Adams ${ }^{9}$. Un fait « dur» relativement à $t$ est décrit par des énoncés dont les conditions de vérité sont entièrement passées à $t$. Exemple : « Théophraste Lupin est né le $1^{\text {er }}$ août 1850 » est un fait dur en 1870. Un fait «souple» relativement à $t$ est décrit par des énoncés dont les conditions de vérité ne sont pas entièrement passées à $t$, et sont donc en partie futures. Exemple: «Le père d'un gentleman cambrioleur est né le $1^{\text {er }}$ août 1850 » est un fait souple en 1870 , sa vérité dépend des actions futures d'Arsène, postérieures à l'année 1870. Si Arsène Lupin devient instituteur à la campagne, il n'altère pas le passé, mais rend fausse la proposition qui décrit ce fait souple... ${ }^{10}$.

La science divine de tel événement futur contingent serait alors un fait souple (soft fact) : un fait qui dépend d'un fait ultérieur (ce que fera, ce qu'aura fait l'agent). Mais alors, «L'agent libre possède une forme de

\footnotetext{
${ }^{8}$ Cf. Alvin Plantinga, “On Ockham's Way Out”, dans Faith and Philosophy, $3: 3$ (July 1986).

${ }^{9}$ McCord Adams, M., «Is the existence of God a « hard» fact?» dans son article de la Philosophical Review vol. $76 \mathrm{n}^{\circ} 4$ (1967), 492-503.

${ }^{10}$ Voir par exemple Daniel J. Hill Divinity and maximal Greatness, Routledge, London \& New York 2005, chapter 3, «Omniscience and Freedom », pp. 69 et suiv. qui résume bien l'esprit des discussions analytiques et déplore avec Hasker la surenchère de termes techniques (p. 95, note 37) : puisqu'on parle de faits «durs », comme pour les œufs, pourquoi pas des faits au plat, des faits brouillés, des faits pochés, des faits bénédictine. Hill réfléchit sur «les conséquences de l'affirmation d'un pouvoir contrefactuel sur le passé, dans le cas un pouvoir sur les croyances divines (ou d'un pouvoir contrefactuel indirect dans le cas des croyances d'un prophète) » (p. 102). Hill estime que la compatibilité de la prescience avec le libre-arbitre réclame que nous ayons un pouvoir contrefactuel sur le passé (en l'occurrence sur les croyances que Dieu aura eu au sujet de nos actions libres postérieurs à ces croyance). Aussi longtemps que la possibilité de l'existence d'un tel pouvoir n'est pas démontrée, Hill considère que cette conception doit être présumée innocente de tout crime logique (p. 103).
} 
pouvoir (contrefactuel) sur les croyances divines passées ${ }^{11}$. Cette notion de pouvoir contrefactuel sur les croyances passées signifie qu'il est au pouvoir d'un agent, à $t$, d'accomplir une action de telle sorte que si cet agent l'accomplit, Dieu n'aura pas eu la connaissance qu'il a eue à to antérieure à t. Car il est au pouvoir de tout agent libre d'agir demain de telle manière que je sache autre chose que ce que je sais aujourd'hui qu'il fera librement. La nécessité du passé s'en trouve compromise : ce qui est fait est fait, ce qui a été cru a été cru. Comment revenir dessus ? Pour concilier la prescience divine avec les actions libres, on est obligé de concéder ce pouvoir d'agir sur le passé. La prescience, pour être infaillible, doit être révisable : ce qui peut fait songer à cet axiome de kremlinologie, à l'heure où l'histoire de la révolution était révisée voire réécrite en fonction des personnalités au pouvoir : « on ne sait jamais aujourd'hui de quoi demain hier sera fait $»$ !

La connaissance de Dieu reste infaillible au prix de sa flexibilité. Que reste-t-il alors de la science divine des futurs contingents libre ? Interrogé sur ce qu'il adviendra (de Ninive, ou du sort de tel apôtre), Dieu devrait répondre : je le sais, mais ce que je sais, je ne pourrai le dire qu'une fois que cela sera arrivé. Alors, je l'aurai su infailliblement. À $t_{0}$, Dieu ne peut pas dire ce qu'il sait infailliblement d'une action contingente libre postérieure à to. À moins de recourir à un subterfuge, comme dans le sketche de Pierre Dac et Francis Blanche ${ }^{12}$, où il ne coûte rien d'attribuer à un devin le pouvoir de dire ce qu'il sait tant qu'il n'est pas sommé d'exercer ce pouvoir.

La parade ockhamiste et son revival analytique ne sont pas donc pas exempts de reproche. La distinction hard facts/soft facts a surtout été mobilisée pour formuler les paradoxes qui résultent d'une science

${ }^{11}$ Cyrille Michon, introd. et trad. Guillaume d'Ockham, Traité sur la prédestination, Paris, Vrin 2007, p. 104. Voir également Prescience et Liberté, Paris, PUF, Epiméthée 2004.

${ }^{12}$ Le Sar Rabindranath Duval : “-Votre Sérénité, pouvez-vous me dire, c'est très important, concentrez-vous, pouvez-vous me dire quel est le numéro du compte en banque de monsieur?

- Oui.

- Vous pouvez le dire?

- Oui!!

- Vous pouvez le dire?

- Oui!!

- Il peut le dire!!! Bravo ! Il est extraordinaire, il est vraiment sensationnel ». 
temporelle de futures contingents libres. Si l'on n'abandonne pas la prémisse de la liberté comme pouvoir d'agir autrement, alors il faudra abandonner la prescience divine des actes contingents libres.

\section{L'alternative atemporaliste}

Considérons l'alternative atemporaliste. Si ce que Dieu sait, il le sait atemporellement, il n'y a plus aucun sens à déclarer que le pouvoir d'agir autrement accordé ou reconnu à un agent a pour conséquence une modification de la science divine. Et même, il nous faut corriger le sens d'expressions comme : à to Dieu sait, dit ou fait quoi que ce soit. Car si l'on prend au sérieux l'atemporalité divine, les modalités temporelles sont improprement rapportées à Dieu.

Il semble bien que la solution atemporaliste résolve le problème ${ }^{13}$. La prescience divine ne risque pas d'être mise en défaut, ou soumise à révision, par un acte libre. Parce qu'elle n'est pas antérieure dans le temps à l'acte en question. Mais tout le monde ne l'entend pas de cette oreille. Hugh Rice objecte : « la conception selon laquelle Dieu est atemporel ne résout pas le problème posé par l'argument de l'incompatibilité, car nous aurions d'aussi bonnes raisons d'affirmer que la science atemporelle de Dieu est fixe $»^{14}$.

A une telle objection, on peut répondre que le concept de fixité est un concept temporel. Il ne s'applique pas à la science divine si celle-ci est atemporelle. Se demander ce que Dieu savait atemporellement avant que A n'accomplisse librement A à t, c'est poser une question sans objet. Un savoir atemporel ne peut être situé avant, après ou pendant un événement temporel. L'atemporalité de la science divine la met à l'abri de toute révision, sans pour autant que nous ayons à considérer que cette science est «fixe» relativement à nous. A proprement parler, pour concevoir la science atemporelle de Dieu, il faudrait que nous puissions attribuer des

${ }^{13}$ Cf. Christopher Hughes : «formidable difficulties beset any account on which God has (infallible) foreknowledge of not yet necessary truths. Accounts on which God timelessly has knowledge of not yet necessary truths avoid at least some of thoise difficulties " "No way Out? " in Reason, Faith and History, Philosophical Essays for Paul Helm, ed. by M.W.F. Stone, Ashgate, Aldershot 2008, pp. 47 et suiv.

${ }^{14}$ Rice, H., "Divine Omniscience, Timelessness and the Power to do Otherwise", dans Religious studies: 42, (123-139), p.134. 
croyances atemporellement. Nous pouvons avoir tendance à interpréter : «Dieu sait atemporellement que A accomplira librement telle action » comme signifiant: "Dieu sait, a toujours su et saura toujours que A accomplit, accomplirait ou a accompli librement telle action ». Mais c'est confondre l'atemporalité avec la sempiternalité. En toute rigueur, ce que Dieu sait atemporellement, il ne le sait jamais à un moment donné. Mais pour nous, à tout moment du temps, nous pouvons affirmer que Dieu le sait (atemporellement). C'est la projection de notre habitude d'indexer temporellement les croyances que nous attribuons (fût-ce « en tout temps ») qui me semble-t-il sous-tend l'objection de Rice, qui confond (peut-être à dessein) intemporalité (validité en tout temps) et atemporalité (validité en dehors du temps). Le fait que nous ne parvenions pas à nous représenter une science atemporelle (dont les propositions n'ont ni date de fabrication, d'emballage ou de péremption, ni même de validité sempiternelle) ne nous autorise pas à la transposer dans le langage temporel, et encore moins à considérer qu'elle est fixée.

D'un autre côté, attribuer à Dieu une science atemporelle d'événements pour nous futurs et contingents nous éloigne de l'appréhension commune de ce qu'est une connaissance. Les tentatives de faire comprendre au moyen d'analogies cette science atemporelle semblent tourner court.

Considérons la célèbre comparaison que propose Boèce de la science divine avec un observateur situé sur le haut d'une colline qui voit défiler l'ensemble d'une colonne en marche, et connaît donc simultanément les positions de l'avant-garde et de l'arrière garde. Selon cette comparaison, le point de vue de Dieu ne diffère que par le degré du point de vue des créatures: sa vision panoramique lui permet de voir plus loin, mais pas l'avenir. Mais ce qui importe dans cette comparaison, c'est que la position de la tête de colonne représente pour les serre-file leur état futur (ou plus exactement un point de passage, une étape ultérieure de leur marche, car l'observateur ne voit pas en même temps le même soldat en deux endroits différents ni à deux moments distincts de sa route). La comparaison serait plus juste si du haut de sa colline, l'observateur voyait simultanément les positions successives du même marcheur. C'est, par hypothèse, le cas pour un observateur atemporel : tous les tubes temporels sont déployés sous son 
« regard ». Regard est à mettre en guillemets puisque Dieu n'assiste pas en spectateur surplombant l'enchainement des événements. Il est plutôt semblable au dessinateur d'une bande dessinée qui a sous les yeux toutes les cases de l'album qu'il conçoit et dessine lui-même (atemporellement).

\section{Ce qui est censé manquer à un Dieu atemporel}

Faute de pouvoir surprendre l'atemporalité divine en flagrant délit d'incohérence logique, on s'en prend à son insuffisance psychologique ou spirituelle. L'atemporalité divine ne rendrait pas justice à ce qu'on attend du Dieu de la révélation. Une foule d'objections a surgi dans la philosophie contemporaine de la religion. Nicholas Wolterstorf est l'un des premiers à lancer l'attaque : «Certaines actions attribuées à Dieu doivent être comprises comme des réponses aux actions libres d'êtres humains - ce que Dieu fait, il le fait parfois en réponse à ce que fait un être humain. Je pense que tel est bien le cas. Et j'estime qu'il s'ensuit, puisque toutes les actions humaines sont temporelles, que les actions de Dieu qui sont des actions "réponses" sont tout autant temporelles $\gg{ }^{15}$. Il sera suivi par Anthony Kenny ${ }^{16}$ et Richard Swinburne ${ }^{17}$. En dépit de quelques contrefeux $^{18}$, c'est le parti temporaliste épaulé par les théologies dites du "process"19 qui s'impose largement. (Mais ce n'est pas parce qu'un parti

\footnotetext{
${ }^{15}$ Wolterstorff, 1975, 'God Everlasting' dans God and the Good: Essays in Honor of Henry Stob, edd. Clifton Orlebeke and Lewis Smedes (Grand Rapids, Mich. Eerdmans), (pp. 181-203), 197 : "Some of God's actions must be understood as a response to the free actions of human beings - that what God does he sometimes does in response to what some human being does. I think this is in fact the case. And I think it follows, given that all human actions are temporal, that those actions of God which are "response" actions are temporal as well".

${ }^{16}$ The God of the Philosophers, Oxford 1979, qui reprend son "Divine Foreknowledge and human freedom" dans le collectif qu'il a lui-même édité Aquinas: A Collection of Critical Essays, London, MacMillan 1969. Kenny conclut (1979: 121) “qu'il est incohérent de supposer que Dieu connaît atemporellement les choses"

17 «God and Time » in Reasoned Faith, edited by Eleonore Stump, Ithaca, N.Y. : Cornell University Press, 1993, pp. 204-22.

${ }^{18}$ Stump, Eleonore, and Norman Kretzmann. « Eternity ». Journal of Philosophy 78 (1981) : 42958. Reprinted in The Concept of God, edited by Thomas V. Morris, pp. 219-52. New York: Oxford University Press, 1987) et Paul Helm (Eternal God : A study of God without Time Oxford, Clarendon 1988)

${ }^{19}$ Les "process theologies" issues de la cosmologie d'Alfred North Whitehead : Process and Reality : An Essay of Cosmology, 1929. Cf. André Gounelle, Le Dynamisme créateur de Dieu. Essai sur la théologie du Process; Paris, Van Dieden editeur, coll. «Références théologiques », 2011. Voir aussi le récent R. T Mullins, The end of the timeless God, Oxford, Oxford University Press 2016.
} 
est majoritaire qu'il a raison, ou tort).

Concentrons-nous sur une objection centrale contre l'atemporalité divine. Considérons, avec Norman Kretzmann, «un film que vous auriez écrit, dirigé, produit, dans lequel vous jouez et que vous auriez visionné des milliers de fois. Vous connaîtriez la moindre scène dans le moindre détail, et vous auriez parfaitement en tête la longueur de chaque scène ainsi que leur enchaînement. [...] Vous connaissez le film incommensurablement mieux que le public au cinéma qui vient le voir pour la première fois. Cependant il y a une chose capitale qu'ils savent et que vous ne savez pas, c'est quelles images sont sur l'écran en ce moment. [...]. Un être omniscient devra non seulement embrasser dans une seule connaissance la séquence intégrale des événements contingents du début à la fin, mais également savoir à quel moment de la séquence on se trouve.... $»^{20}$

Cette comparaison inspirera Anthony Kenny qui reprend à Arthur Prior l'objection - ou la caricature - d'un Dieu incapable de comprendre ce que c'est que d'attendre le résultat d'un examen (académique ou médical!) ${ }^{21}$. Un Dieu atemporel passerait donc à côté de l'essentiel: ce que nous vivons au moment où nous le vivons. Un Dieu qui manque sacrément d'empathie!

Cette comparaison en forme d'objection ne reste pas sans réponse. Car la science atemporelle de Dieu est aussi une science des états mentaux de chaque créature. On suppose donc que Dieu a accès à la connaissance de la situation temporelle de chacune, et sait qu'à tel moment de sa trajectoire, il

\footnotetext{
${ }^{20}$ N. Kretzmann, 'Omniscience and Immutability', Journal of Philosophy, July 1966, p. 414 : “the knowledge you might have of a movie you had written, directed, produced, starred in and seen a thousand times. You would know its every scene in flawless detail, and you would have the length of each scene and the sequence of scenes perfectly in mind. (...) You know the movie immeasurably better than do the people in the theatre who are now seeing it for the first time, but they know one big thing about it you don't know, namely, what is now going on on the screen. Thus, the similar account of omniscience regarding contingent events is drastically incomplete. An omniscient being must know not only the entire scheme of contingent events from beginning to end at once, but also at which stage of realization that scheme is now".

21 «Dieu serait incapable [...] de savoir si la session des examens finaux de 1960 à Manchester est à présent close. En effet ce n'est pas là quelque chose que Lui ou quiconque pourrait savoir atemporellement, tout simplement parce que ce n'est pas une chose qui est vraie atemporellement » " The Formalities of Omniscience » dans Arthur Prior, Papers on Time and Tense (London, Oxford University Press, 1968), p. 29.
} 
lui reste tel et tel événement à découvrir. De sorte que loin d'ignorer la réponse à la question : " où en est le film projeté actuellement ? », Dieu est - atemporellement - en mesure de décrire la perspective temporelle de l'attente de tel spectateur qui découvre tels et tels événements contingents. Une science atemporelle n'est pas une ignorance des modalités temporelles. Ce n'est pas le contenu de cette science qui est atemporel, c'est son statut d'événement. «Dieu sait... » ne se conjugue ni au présent, ni au passé, ni au futur. Mais rien n'empêche que ce que Dieu sait puisse avoir trait à une succession d'événements: Dieu sait atemporellement qu'en 2018 je n'ai pas encore le diagnostic médical que j'attends avec impatience. Nelson Pike niait pour sa part que des propositions qu'un être atemporel fût condamné à ignorer les faits rapportés par des propositions comme «c'est la première scène qui passe en ce moment à l'écran » ou « Aujourd'hui nous sommes le 12 mai », soit qu'il ne s'agisse pas de faits, soit parce qu'il est possible de les désigner au moyen d'expressions dénuées d'indexicaux temporels. Selon Pike, « tout ce qu'on aura montré, c'est qu'il y a certaines formes verbales qu'un être atemporel ne saurait utiliser s'agissant de formuler ou de rapporter sa connaissance $\gg^{22}$.

\section{$V$. Le molinisme : no other game in the town?}

La solution moliniste offre un bel exemple d'exhumation de la scolasique par la philosophie analytique de la religion. Très prisée dans les universités américaines $^{23}$, cette solution a la part belle dans les débats contemporains. C'est Robert Merryew Adams qui a introduit en 1977 le débat sur la connaissance moyenne dans la philosophie analytique ${ }^{24}$. Adams estime que le contenu propositionnel d'un contrefactuel de la liberté (ce que ferait mon boucher si je lui demandais de me vendre de la viande qu'il a sur son étalage) reste probable (probablement il me la vendrait). La connaissance

\footnotetext{
22 « all that has been established is that there are certain forms of words that a timeless individual could not use when formulating or reporting his knowledge. » (Pike, 1970 : 95). Mais Pike affirme que l'atemporalité est incompatible avec la création ou la conservation du monde ou encore avec la conception d'un Dieu personnel.

${ }^{23}$ Alfredo Freddoso a donné une traduction de la IVème partie du Liberi Arbitrii cum Gratiae Donis, Divina Praescientia, Providentia, Praedestinatione et Reprobatione Concordia, en 1988 : On Divine Foreknowledge: Part IV of the Concordia, Ithaca: Cornell.

${ }^{24}$ « Middle Knowledge and the Problem of Evil », American Philosophical Quarterly 14: 109-117.
} 
des contrefactuels ne permet pas à Dieu d'opérer un choix qui sauve sa prescience en même temps que la liberté. Thomas Flint, qui 1 a consacré au molinisme un ouvrage de référence, plaisante à son sujet : «c'est la seule distraction en ville (there is no other game in town) $\rangle^{25}$. Molina attribue à Dieu une science moyenne de ce que A ferait/aurait fait en telle ou telle circonstance $^{26}$. Dieu compare tous les scénarios possibles choisit celui (ou l'un de ceux) où son dessein se réalise. Il crée un monde où les créatures ne manqueront pas d'accomplir ce qu'elles accomplissent. Et cependant, il est concevable qu'elles auraient pu agir autrement. Solution qui permet de conjuguer l'accès de Dieu aux actions futures, qui sont déterminées (ou infaillibles), avec la liberté de certaines et la contingence de certaines d'entre elles, sans avoir à mobiliser le fameux pouvoir contrefactuel sur le passé.

En dépit de sa virtuosité technique, Molina ne nous permet pas d'attribuer dans ce monde créé un véritable pouvoir d'agir autrement. Sans doute, la liberté de l'agent est attestée dans les mondes possibles. Mais dans ce monde actuellement existant, un seul scénario est possible. Le molinisme présuppose qu'on puisse donner un sens aux «contrefactuels de la liberté », et que ces contrefactuels (les réponses à la question « qu'aurait fait Napoléon s'il avait gagné Waterloo ?») sont antécédents à toute détermination par une volonté divine ${ }^{27}$.

Franciscains et jésuites se sont donc évertués à trouver une solution : la solution dominicaine (inspirée de Boèce) me semble de loin préférable. Bien sûr, l'essentiel de la solution proposée par Boèce puis Thomas sur la question de savoir si la science divine des futurs supprime, le cas échéant, la contingence de ces futurs, est dans la remarque qu'il n'y a pas transmission de la nécessité de cette science divine. Si Dieu sait qu'il y

${ }^{25}$ CF Thomas P. Flint, Divine Providence : The Molinist Account, Cornell University Press, Ithaca and London, 1998, p. 75.

${ }^{26}$ William Hasker, dans God, Time, and Knowledge, Ithaca: Cornell (1998) a proposé une critique assez complète de la science moyenne des molinistes.

27 Thomas P. Flint, Divine Providence : The Molinist Account, Cornell University Press, Ithaca and London, 1998, p. 76. Flint expédie un peu vite «The Eternity Alternative» (pp. 82-84) et plus longuement «The Thomist Alternative » (pp. 85-94) qui selon lui prête le flanc à l'objection du mal. Plusieurs philosophes semblent reprocher au thomisme une détermination complète de tout événement par Dieu, cause première qui concourt à l'action de tout agent. 
aura une bataille navale, alors nécessairement il y aura une bataille navale, mais c'est la conséquence qui est nécessaire, pas le conséquent. Ainsi, si Dieu sait ce que je ferai librement demain, alors nécessairement c'est cela que je ferai demain, mais librement. La modalité contingente de l'action n'est pas en tant que telle compromise par la nécessité d'une science infaillible.

\section{Le problème $d u$ " possible prophet »}

Avant de revenir sur l'intérêt de cette solution atemporaliste de la science divine des futurs libres, nous pouvons évoquer un problème rencontré par la littérature contemporaine en philosophie analytique de la religion. C'est le problème de la prophétie possible. Si Dieu sait atemporellement ce que A accomplit à $t$, rien n'empêche que Dieu fasse en sorte (atemporellement) que soit révélé à to ce que $\mathrm{A}$ accomplira à t postérieur à to... Auquel cas $\mathrm{A}$ n'est plus libre de ne pas l'accomplir, sous peine d'invalider la prophétie. On peut alors redéclencher l'argument de l'incompatibilité sous la présupposition de la science atemporelle de Dieu ${ }^{28}$.

Nous serions revenus au point mort. Un Dieu atemporel peut en effet révéler la venue d'un événement contingent futur (non par rapport à Dieu, mais par rapport à l'instant où il est révélé.

On peut ici s'inspirer d'une réponse de Swinburne aux paradoxes de la toute-puissance. Discutant le paradoxe de la pierre dû à Wade Savage (Dieu peut-il créer une pierre qu'il ne peut pas soulever?) Swinburne écarte la solution facile qui consiste à affirmer que « une pierre que Dieu ne peut pas soulever» ne peut pas exister, et que par conséquent ne pas pouvoir créer cette pierre (qui ne saurait exister) ne limite pas sa toutepuissance. Swinburne argue que si Dieu exerce effectivement (par opposition à : détient simplement le pouvoir) sa capacité de produire l'existence d'une pierre trop lourde pour qu'il puisse par la suite procéder à son soulèvement, alors il cessera d'être tout-puissant. Mais libre à Dieu

28 David Widerker, "A Problem for the Eternity Solution", International Journal for the Philosophy of Religion, vol. 29 (1991), 87-95, et la réponse d'Eleonor Stump dans "Prophecy, Past truth, and Eternity", dans Philosophical Perspectives vol. 5, Philosophy of Religion, (1991) pp. 395-424. 
de rester omnipotent en n'exerçant pas ce pouvoir de créer des pierres trop lourdes à soulever ${ }^{29}$ !

De même, dans le cas de la prophétie, on pourrait soutenir que si toutes les actions sont atemporellement présentes à Dieu, même celles que l'action libre des créatures produira, rien n'empêche que Dieu ne puisse révéler une action avant qu'elle ne se produise. Mais le fait-il ? On pourrait dire qu'il peut le faire, mais n'est pas pour autant tenu de le faire. Car s'il le faisait, alors on pourrait nier qu'il soit réellement au pouvoir de la créature, dont l'action est prophétisée, d'agir autrement. A moins que Dieu ne réserve de telles prophéties qu'à des cas où la liberté s'exerce sans pouvoir d'agir autrement. Car au fond, s'il semble raisonnable de défendre une conception «libertarienne» de la liberté, pourquoi cette conception devrait-elle être exclusive? Certains actes seraient dits libres du fait que l'agent a le pouvoir d'agir autrement. D'autres resteraient libres mais seraient tels qu'il n'est pas au pouvoir de l'agent d'agir autrement. Supposons qu'un ange envoyé de Dieu vienne annoncer à une jeune fille de Nazareth qu'elle va enfanter le Messie. Il importe que son fiat, le cas échéant, exprime un consentement libre, mais s'il est possible à la jeune fille en question d'agir autrement, alors il faut que l'annonciation soit accompagnée d'un plan B, en cas de refus (en cas de non fiat, ou plutôt non serviam). Il faut alors imaginer, comme l'a fait Vladimir Volkoff dans une amusante nouvelle des Chroniques angéliques (Paris, Fallois - l'Age d'Homme, 1998) un ange Gabriel faisant du porte-à-porte, jusqu'à ce qu'il tombe sur la bonne personne. (A dire vrai, la théorie moliniste recourt elle aussi à ce porte-à-porte de l'ange Gabriel, explorant les réactions mais dans les mondes possibles. Dans un monde, la jeune fille répond : «non », dans un autre, elle répond : « oui mais pas tout de suite », dans un énième, elle (ou une autre ?) répond: "qu'il me soit fait selon ta parole», et c'est ce monde que Dieu rend actuel). Or la nativité ainsi annoncée fait l'objet de prophéties, et suppose certains préparatifs et adjuvants (trouver un mari qui accepte de prendre la jeune fille enceinte comme épouse, lui évitant d'être lapidée, faire promulguer un édit de recensement qui entraîne le père adoptif à se rendre au chef-lieu de son clan d'origine, etc.). L'explication

\footnotetext{
${ }^{29}$ R. Swinburne, The coherence of theism, Oxford University Press, 1993, chapter 9, pp. 153-163.
} 
la plus simple est que certaines prophéties concernent des actions déterminées et, le cas échéant, dans lesquelles la liberté de l'agent n'est pas du type d'un pouvoir d'agir autrement. Autrement dit, Dieu ne révélera à l'avance que des événements dans lesquels la liberté est de type compatibiliste... Rappelons néanmoins que l'expression «à l'avance» ne décrit pas la science divine, mais la présence dans le temps humain d'une prophétie portant sur un acte encore à venir.

\section{Le modèle de la connaissance pratique}

Supposé réglé le problème, une question reste en suspens : comment Dieu peut-il avoir la science des futurs contingents libres? La réponse d'Anscombe s'inspire de Thomas (ST II-II, q. 33, a.1c). Elle oppose "la raison spéculative qui se contente d'appréhender des réalités [...] à la raison pratique qui non seulement les appréhende mais les cause ${ }^{30}$.

C'est ce concept de connaissance non-observationnelle, qu'Anscombe compare d'abord avec la connaissance qu'on a de la position de nos membres même les yeux fermés (even with your eyes shut), et sans qu'on dispose de sensations séparément descriptibles qui produiraient cette connaissance (Intention, pp. 13 et 15). Anscombe recourt aussi à une autre comparaison : la connaissance qu'a un chef de chantier qui dirigerait à distance la construction d'un immeuble sans voir ni entendre aucun retour sur le progrès des travaux (p. 82), la connaissance qu'on a de ce qu'on écrit même quand on ferme les yeux (p. 53), la liste de commissions d'un acheteur au supermarché, par opposition avce la liste que dresserait le détective qui suivrait à la trace l'acheteur (p. 56), enfin la connaissance que Dieu a de la création (p. 87). Knowing by doing en quelque sorte. On peut noter que dans tous les cas évoqués, la connaissance pratique est simultanée avec l'activité ou la situation connue. Dans le cas qui nous occupe, il faudrait concevoir que Dieu connaît pratiquement ce qu'il fait atemporellement (créer et soutenir dans l'existence toute la réalité), action atemporelle qui a des effets temporels (le déroulement des événements de l'univers).

\footnotetext{
30 "speculative reason only apprehends things, [...] practical reason not only apprehends but also causes them" (Intention, Oxford: Basil Blackwell, 1957, p. 53).
} 
La connaissance qu'a Dieu de ce que font librement les créatures serait donc une connaissance pratique, atemporelle. Il n'y a aucune nécessité que la connaissance qu'on a d'une action temporelle soit elle-même temporelle, comme on l'a remarqué plus haut. Comme le suggère William Hasker, affirmer que « la manière dont Dieu connaît l'existence des choses est la manière dont les choses existent vraiment » n'implique pas que «la manière dont Dieu connaît les réalités ( sa façon de les connaître) soit la même que leur façon d'exister $»^{31}$.

Dans le cadre de la redécouverte de la notion de connaissance pratique, on dira que Dieu sait ce que font les créatures librement, parce que c'est lui qui opère en tout agir des créatures. Mais alors que reste-t-il aux créatures, sont-elles de simples marionnettes ? Cette question est rendue plus aigüe par la compréhension de la création, comme création continuée.

\section{La création continuée et le soupçon d'occasionnalisme}

C'est Harry Frankfurt qui a, sinon relancé, du moins popularisé le débat sur la création continuée, en introduisant la notion (qu'il récuse) d'inertie ontologique. Le monde, une fois créé, n'est pas doué d'inertie ontologique! «Les réalités créées, outre qu'elles doivent à Dieu leur création, lui doivent à chaque moment la continuation de leur existence. Elles ne peuvent pas plus persister sans Dieu qu'elles ne peuvent commencer d'exister sans lui $\gg{ }^{32}$. C'est ainsi que la philosophie analytique contemporaine redécouvre Boèce de Dacie et Thomas: "Dieu ne se contente pas de donner à ses créatures des pouvoirs et des dispositions de départ pour ensuite, comme la divinité des déistes, retourner s'asseoir (sit back) et laisser les réalités évoluer toutes seules. Pas davantage son contrôle ne s'étend qu'à certains traits du monde, tandis que le détail lui

\footnotetext{
31 " The way God knows things to be is the way things really are » does not entail « The way in which God knows things (i.e. his manner of knowing them) is the same as the way in which they exist », Pike, N., God, Time, and Knowledge (Ithaca, N.Y.: Cornell University Press) 1989, p. 166

${ }^{32}$ Frankfurt, H., Necessity, Volition and Love, Cambridge University Press, 1998, dans le chapitre 5 intitulé "Continuous creation, ontological inertia and the Discontinuity of Time”, p. 55.
} 
échapperait. [...] Chaque événement, quelle que soit son ampleur, est sous contrôle de Dieu, et intégré à son plan général pour le monde $»^{33}$.

Timothy Miller formule deux objections contre la continous creation: " $1^{\circ}$ ) elle est incompatible avec la persistance des réalités créées, $2^{\circ}$ ) elle est incompatible avec la causalité seconde" ${ }^{34}$. Bref, on soupçonne cette doctrine d'avoir partie liée avec une version ou une autre de l'occasionalisme. Mais qu'entend-on ici par occasionalisme? Alfredo Freddoso définit l'occasionnalisme en ces termes : «Dieu seul est cause d'effets dans la nature; et contrairement à l'opinion commune, les substances naturelles n'apportent pas la moindre véritable contribution causale à ces effets; les substances créées sont incapables d'action transitive, i.e. d'actions ayant des effets extérieurs à l'agent $»^{35}$. Ou, pour parler avec Daniel Lim : «Dieu est l'unique entité qui soit causalement efficace dans le monde», «l'activité causale des créatures ne se produit jamais $\gg .{ }^{36}$

C'est encore le soupçon d'Andrew Pessin: «Lorsque Dieu crée (continûment) ou conserve des substances, il semble bien qu'il faut qu'il les crée de façon déterminée, avec tous leurs modes et leurs caractéristiques ; or cela paraît impliquer que la responsabilité causale de toutes ces caractéristiques incombe à Dieu seul, ne laissant pas aux créatures la moindre contribution ${ }^{37}$.

\footnotetext{
${ }^{33}$ Flint, T. (1998: 13). Flint rappelle que c'est la conception défendue par la confession de Westminster de 1647, par Vatican I, et déjà dans l'Agamemmnon d'Eschyle (1998 : 15, 20).

${ }^{34}$ Miller, Timothy D., "Continuous creation and secondary causation: the threat of occasionalism", Religious Studies (2011) 47, (3-22), p. 3. Comme le remarque Miller, Philipp Quinn estime que "la création continuée peut-être conjointe avec une théorie régulariste, contrefactualiste ou nécessitariste de la causalité des causes secondes" sans impliquer l'occasionnalisme (p. 11).("CC can be consistently conjoined with regularity, counterfactual and necessitarian theories of secondary causation").

${ }^{35}$ Freddoso, A.J. "God's General Concurrence with Secondary Causes: Why Conservation is not Enough," Philosophical Perspectives, 5 ed. James E. Tomberlin (Atascadero, CA: Ridgeview Publishing, 1991), (553-585), p. 554 : "God alone causes effects in nature; natural substances, contrary to common opinion, make no genuine causal contribution at all to any such effect. In short, there is no creaturely or "secondary" causation in nature; created substances are incapable of transeunt action, i.e., action that has effects outside the agent".

${ }^{36}$ Daniel Lim, dans God and Mental causation, Spingerbriefs, 2015, p. 22 : "God is the only causally efficacious entity in the world" ou encore "creaturely causation never occurs".

37 Andrew Pessin, "Does Continuous Creation entail occasionalism?", Canadian Journal of Philosophy, Vol. 30, n 3, Sep. 2000, (413-439), p. 418. Selon Pessin : "la création continue -la thèse que Dieu maintient toutes les substances dans l'existence (that God sustains all substances)
} 
Leibniz reconnaissait déjà par endroits que certaines conceptions de la CC semblent refuser l'existence ou l'opération autonome des substances créées : « la conservation est une création continuée. En conséquence de cette doctrine, il semble que la Créature n'existe jamais $»^{38}$.Est-ce par coquetterie que Leibniz agite ici une sorte d'épouvantail occasionaliste? C'est ainsi que l'ont entendu beaucoup de philosophes analytiques. Selon Jonathan Kvanvig : «Nous avons là un des rares lieux philosophiques où nous pouvons trouver une quasi-unanimité d'opinion: quoiqu'on veuille dire au sujet de la relation entre Dieu et l'ordre du créé, nous ne devrions pas exprimer de sentiments occasionnalistes ${ }^{39}$.

Comme l'écrit Swinburne, «Le théisme n'est certainement pas tenu à la position occasionnaliste (que je juge manifestement fausse) selon laquelle les conditions physiques ou les substances ne produisent jamais d'effets, mais que tous les effets sont produits par des agents rationnels, en l'occurrence par Dieu. L'occasionnaliste affirme que la mise à feu de la poudre ne provoque pas réellement d'explosion; c'est seulement que, à chaque mise à feu, Dieu cause l'explosion. La position beaucoup plus plausible que je défends est que Dieu cause le fonctionnement de la causalité scientifique ; c'est lui qui est responsable du fait que la mise à feu de la poudre cause une explosion.

Comment comprendre que Dieu soit cause que la poudre provoque une explosion (God causes the gunpowder to cause explosion)? En quel sens Dieu fait faire quelque chose à la poudre ? Comment la causalité naturelle est-elle nécessaire si son fonctionnement est suspendu à une opération divine ».

est susceptible de conduire à l'occasionnalisme, mais seulement si on lui ajoute deux prémisses : (i) que Dieu explicitement veuille et determine tous les modes et (ii) qu'il n'y ait pas de surdétermination" ( p. 433).

${ }^{38}$ Leibniz, Sämtliche Schriften und Briefe, hrsg. von der Berliner Brandenburgischen Akademie der Wissenschaften, 2001, Band VI, 4, C (Philosophia naturae), p. 2319. Leibniz 1978 (1710) IIIème Partie, $\S 382$, et $\S 386$ (342) Et dans son Rationale fidei catholicae, Leibniz souligne la dépendance perpétuelle et absolue des réalités par rapport à Dieu (res semper in existendo absolute a Deo dependent aeque in progressu ac initio), et du même coup leur renouvellement permament, leur reproduction intégrale (nihil dici potest ex praecedenti restare quod non et ipsum denuo producatur)

${ }^{39}$ Kvanvig, J. "Creation and Conservation", The Stanford Encyclopedia of Philosophy (Fall 2008 Edition), Edward N. Zalta (ed.), URL =

$<$ http://plato.stanford.edu/archives/fall2008/entries/creation-conservation/> (2008) 
Dans la même note de bas de page, Swinburne énonce une autre raison de refuser l'occasionnalisme : "Non seulement l'occasionnalisme semble heurter de front une donnée manifeste de l'expérience - à savoir que les objets physiques (ou leurs états) sont souvent la cause d'événements ; mais il serait suicidaire, pour le théiste qui veut conclure de l'univers physique et de ses caractéristiques à Dieu, de nier que les objets physiques soient jamais causes d'événements. En effet notre conception des critères de causalité est dérivée, par extrapolation, d'innombrables situations intramondaines où il nous semble bien que tel objet physique est la cause de tel événement. Mais si, en réalité, il n'y a pas, dans ces situations, une causalité de ce type, alors nos critères d'identification de la causalité nous mettent sur une fausse piste, et il sera erroné de les utiliser pour conclure que Dieu est la cause de l'existence de l'univers ou de quoi que ce soit d'autre $\gg^{40}$. Voilà une raison pas banale de récuser l'occasionnalisme.

\section{Un remède préventif : une division ontologique du travail ?}

Jonathan Kvanvig a proposé de prévenir tout risque d'occasionnalisme en recourant à une véritable division ontologique du travail : «en développant une métaphysique dans laquelle les objets sont ontologiquement fondamentaux, tandis que les événements, les processus, les faits et les états de choses sont des entités dérivées [...], nous faisons naître l'espoir de pouvoir expliquer pourquoi la dépendance ontologique s'applique sélectivement plutôt qu'universellement, et donc l'espoir de garantir aux causes secondes une place dans la nature de façon à expliquer les séquences d'événements tout en conjurant le spectre de l'occasionalisme ». La création continuée porterait exclusivement sur les substances, qui opèreraient sans le concours de la cause de leur existence. Dans le même sens, Miller recommande d'adopter un concurrentisme faible, dans lequel « Dieu peut causer l'existence des individus sans causer tous leurs états ou toutes leurs propriétés $»^{41}$. Ce disant, on évite semble-t-

\footnotetext{
${ }^{40}$ Swinburne R., The existence of God, 2nd ed. Oxford Univresity Press, 2004, pp. 107-108, note de bas de page 10 .

${ }^{41}$ Miller, Timothy D., "Continuous creation and secondary causation: the threat of occasionalism", Religious Studies (2011) 47, (3-22), p. 10.
} 
il, la surdétermination, on garantit aux causes secondes leur rang, dans une sage subsidiarité (de minimis non curat preator).

Cette division du travail pose plusieurs problèmes.

Si l'opération des causes secondes échappe à Dieu, quel accès a-t-il à la connaissance du cours des événements? L'opération spontanée des créatures risque d'être totalement désordonnée, chaotique. L'opération divine dans les causes secondes faisait double-emploi (c'était le risque de surdétermination causale), son absence risque de faire désordre. Cette division du travail ontologique se heurte d'ailleurs à forte partie. Songeons à la position de Thomas selon laquelle Dieu est en tout agent la cause de son agir : " Ainsi donc Dieu est cause de n'importe quelle action dans la mesure où il donne le pouvoir d'agir, et dans la mesure où il le conserve, où il l'applique à l'action et où tout autre pouvoir agit par le sien. Et comme nous avons ajouté à cela que Dieu est son propre pouvoir, et qu'il est en n'importe quelle chose, non comme une partie de son essence, mais comme celui qui la conserve dans l'être, il s'ensuit que lui-même opère sans intermédiaire en n'importe quel être en son opération, sans exclure l'opération de la volonté et de la nature $»^{42}$.

Lorsqu'un cuisinier cuit un poulet, c'est le feu qui rôtit de poulet, mais on dit bien que c'est le cuisinier qui cuit le poulet (et on lui imputera une cuisson parfaite, trop brève ou trop longue). Ainsi, toute application d'un pouvoir à une action quelconque est principalement et premièrement de Dieu. Cette doctrine se maintient au moins jusqu'à Théophraste Bouju : «Encores que Dieu apres auoir creé les chofes, les ait laiffees aux forces de la nature \& à fon cours : c'eft néantmoins en telle forte qu'il y affifte toufiours, qu'il coopere auec elles, leur inflüe l'eftre, les conferue $\&$ en a la

\footnotetext{
${ }^{42}$ De Potentia, Qu. 3, art. 7, corpus: "Sic ergo Deus est causa actionis cuiuslibet in quantum dat virtutem agendi, et in quantum conservat eam, et in quantum applicat actioni, et in quantum eius virtute omnis alia virtus agit. Et cum coniunxerimus his, quod Deus sit sua virtus, et quod sit intra rem quamlibet non sicut pars essentiae, sed sicut tenens rem in esse, sequetur quod ipse in quolibet operante immediate operetur, non exclusa operatione voluntatis et naturae ». Voir aussi Contra Gentes, III, 67 : «Or de même que Dieu n'a pas donné l'être aux choses seulement à leur début, mais qu'il cause l'être en elles tant qu'elles sont en les conservant dans l'être - on l'a montré - de même il ne leur a pas donné leur vertu seulement au premier temps de leur création, mais il la cause toujours en elles. Aussi tout influx divin se retirant, toute activité tomberait. Toute opération ressortit donc à Dieu comme à sa cause ».
} 
pouruoyãnce \& le gouvernement 》. De même : «Dieu opere de forte és chofes, qu'elles ont leur propre operation $»^{43}$.

On pourrait aussi convoquer ici la doctrine de la prémotion divine, qui décrit un concours instrumental de Dieu contre la coneption d'une la coopération indépendante de la part de causes seconds. David Oderberg l'a tout récemment remise à l'honneur et défendue avec panache ${ }^{44}$

La prémotion est le mouvement reçu dans la créature, mouvement naturel (et non moral ou final), ce mouvement est premier, non temporellement mais dans la hiérarchie des causes; il est prédéterminant : la cause seconde accomplit infailliblement ce que Dieu la conduit à accomplir. Les causes secondes sont ainsi des instruments de la cause première. Dieu « instrumentalise» les causes secondes. Dieu est le Maître souverain de son dessein. Mais pour sa réalisation, Il se sert aussi du concours des créatures.

C'est bien le couteau qui coupe le pain mais ce pouvoir sécant, il ne l'exerce que parce que je l'utilise comme instrument. L'instrument a deux opérations : celle qui est due à sa forme (le tranchant du couteau) et celle qui est due à sa mobilisation par un agent ${ }^{45}$.

Selon David Oderberg, cette doctrine est la seule qui respecte à la fois la réalité des causes secondes et le concours exhaustif de Dieu à toute action

43 Théophraste Bouju, Corps de toute la philosophie, à Paris, chez Denys de la Noüe, 1614, Métaphysique particulière, Livre I, chapitre XLVII, pp. 953 et 960.

${ }^{44}$ David S. Oderberg, "Divine premotion”, International Journal for Philosophy of religion, June 2016, Vol 79, Issue 3, pp. 207-222.

45 "secundum quod est motum a per se agente, De Veritate q.27 a.4, resp. Cf. Catéchisme romain Prima Pars, Capitulum II, 22, p. 31: "Deus sua gubernatione secundarum causarum vim non evertit. Non solum autem Deus universa quae sunt providentia sua tuetur atque administrat, verum etiam quae moventur et agunt aliquid, intima virtute ad motum atque actionem ita impellit, ut, quamvis secundarum causarum efficientiam non impediat, praeveniat tamen, cum eius occultissima vis ad singula pertineat, et quemadmodum Sapiens testatur: Attingat a fine usque ad finem fortiter, et disponat omnia suaviter. Quare ab Apostolo dictum est, cum apud athenienses annuntiaret Deum quem ignorantes colebant: Nonlonge est ab unoquoque nostrum; in ipso enim vivimus, et move mur, et sumus" Non seulement Dieu, par sa Providence, soutient et gouverne toute la création; mais c'est Lui qui en réalité communique le mouvement et l'action à tout ce qui se meut et à tout ce qui agit; et de telle sorte qu'Il prévient, sans l'empêcher, l'influence des causes secondes".

De même encore, dans le catéchisme de 1992 : "Dieu agit en tout agir de ses créatures. Il est la cause première qui opère dans et par les causes secondes : " Car c'est Dieu qui opère en nous à la fois le vouloir et l'opération même, au profit de ses bienveillants desseins " (Ph 2, 13 ; cf. 1 Co 12, 6) » (CEC, 306). 
(the exceptionless nature of divine concurrence). Mais alors comment dissiper le soupçon d'occasionalisme, qui réduit les causes secondes à des figurants sans efficace causale?

\section{L'atemporalité de la création dissipe les soupçons d'occasionnalisme}

Je voudrais montrer que la création, pas seulement restreinte aux substances mais étendue à leurs opérations, n'annule pas leur contribution causale. La conception relationnelle et atemporelle de la création promue par Thomas est à même de dissiper ce soupçon.

Ce n'est pas de la même manière que Dieu produit le déroulement des causes secondes et que celles-ci produisent leur effet propre. Les causes secondes produisent temporellement leur effet propre parce qu'elles sont dotées atemporellement de pouvoirs et de dispositions par Dieu.

Si l'on adopte la conception d'un Dieu atemporel, qui opère atemporellement (operari sequitur esse), le concours de Dieu non seulement à toutes les existences mais encore à toutes les actions ne détruit pas la causalité seconde : «Dieu a créé toutes choses sans intermédiaire, mais il a dans la création elle-même des réalités instauré un ordre dans les réalités, tel que les unes dépendissent des autres, et fussent par elles conservées dans l'existence, à titre secondaire ; étant toujours présupposée la conservation principale, qui se fait par Dieu lui-même $»{ }^{46}$.

La réalité des pouvoirs naturels et l'efficace des causes secondes est portée par l'opération conservatrice principale. La dépendance causale des causes secondes est réelle. Même si c'est Dieu qui institue dans ces réalités un ordre, et qui utilise les causes secondes pour les conduire à leurs

\footnotetext{
46 "Deus immediate omnia creavit, sed in ipsa rerum creatione ordinem in rebus instituit, ut quaedam ab aliis dependerent, per quas secundario conservarentur in esse; praesupposita tamen principali conservatione, quae est ab ipso », Summa Theologiae I, q. 104, art. 2, ad 1. Cf Suarez : " Dieu opère per se ac immediate en toutes les opérations de ses créatures, , et cette influence (hunc influxum ejus) ets absolument necessaire, pour que la creature réalise (efficiat) quoi que ce soit Disputatio XXII, De prima causa, et alia ejus actione, quae est cooperatio, seu concursus cum causis secundis, Sectio I, An possit sufficienter probari ratione naturali, Deum per se ac immediate operari in actionibus omnium creaturarum, $6 \&$ 7, Suarez, Opera omnia, Paris, Vivès MDCCCLXI, t. 25 , pp. $803 \mathrm{a}$ and b.
} 
opérations. Mais comment dissiper tout soupçon d'occasionnalisme ou de surdétermination causale?

La meilleure garantie de la non redondance des causalités créatrice et naturelles réside dans la non-temporalité de la première et la temporalité de la seconde. Dans la conception atemporaliste, la création n'est ni un changement, ni un mouvement, ni un événement. Elle n'est pas l'activité de transformation du néant en quelque chose. C'est seulement métaphoriquement que nous nous représentons qu'une réalité possède l'existence après la non-existence (creatum habere esse post non-esse).

Si Dieu opère atemporellement, la notion de création continuée se réfère à une continuation d'existence du côté de la créature et non d'une poursuite dans le temps de l'effort divin; (la simplicité divine exclut que Dieu ait des parties temporelles)

C'est la conception défendue bec et ongles par Sertillanges, qui développe d'abord la reprise cartésienne de cette doctrine. Le concept de création continuée ne fait pas dépendre l'action divine du temps des créatures : " quand il [Descartes] parle de création continuée, il ne peut pas vouloir dire que Dieu, lui, continue à créer, puisqu'il crée intemporellement, par une action qui ne se distingue pas de lui-même, et que pour continuer il faut être dans le temps $»^{47}$; « [...] la création est un acte intemporel en ce qui concerne Dieu, et $[\ldots]$ dans la créature elle est pure relation, intemporelle ainsi en elle-même [...] La création ne continue pas ; ce sont les choses qui continuent et la création pose leur durée avec elles $»^{48}$.

C'est Dieu qui, atemporellement, fait que les substances contribuent causalement à ce qui arrive. Les causes secondes opèrent d'elles-mêmes, mais pas seules : c'est de Dieu qu'elles tiennent leur pouvoir d'opérer par elles-mêmes, et elles sont l'instrument de son opération.

$\mathrm{Si}$ au contraire on se range à une conception temporaliste de la création divine, alors nous avons deux causes suffisantes d'un effet donné qui opèrent simultanément. Si l'on disait qu'à $t_{1}$, Dieu fait exister et agir $x$ par lui-même à $t_{1}$ ou à $t_{2}$ (préordination des causes) il y aurait concurrence (et

\footnotetext{
${ }^{47}$ A.-D. Sertillanges, L'idée de création et ses retentissements dans la philosophie, Paris, Aubier, 1945, p. 74.

${ }^{48}$ Ibid., pp. 162-163.
} 
pas concours) entre deux causes suffisantes par soi. L'une d'entre elles est superflue.

En revanche, un créateur opérant atemporellement peut être causalement responsable de l'opération néanmoins autonome de ses créatures. Le créateur n'exerce pas le pouvoir causal des créatures à leur place : il leur donne le temps pour le faire, dans lequel il n'agit pas. Elles exercent temporellement un pouvoir qu'il leur confère atemporellement.

La conception atemporelle de la création continue de Dieu garantit sa souveraineté absolue en préservant l'autonomie des causes secondes. Dès lors que faire exister et faire agir quelque réalité dans le temps est une opération atemporelle, elle ne démet pas de son opération propre la réalité temporelle.

\section{$X$. Excursus romanesque}

$\mathrm{Au}$ moment de clore cette évocation fleuve des reformulations contemporaines du problème de la prescience divine et de la liberté des créatures, faisons une pose littéraire. Nous ne disposons pas d'analogie ou d'image d'une telle structure causale à double temporalité. On pourrait à la limite suggérer, avec beaucoup de réserves, la comparaison suivante :

Soit un romancier intégralement responsable des personnages et de l'intrigue de son roman. Tout ce qui s'y passe, actions délibérées ou événements déterministes, dépend de sa décision d'auteur. Le casting et le scénario sont de bout en bout dépendants de lui.

Toutefois, si nous lisons dans le roman que « la marquise sortit à cinq heures », l'explication du fait sera susceptible de deux registres. Il y aura le registre interne au roman (les personnages, leurs habitudes sociales ou leur libre décision etc) : c'est le niveau de l'autonomie temporelle des causes secondes. Si le roman est bien écrit, on n'ira pas répondre que si la marquise sort à cinq heures, c'est parce que tel est le bon plaisir du romancier. Mais une réponse tout aussi valable sera: c'est parce que l'auteur l'a écrit (registre externe). C'est, pour ainsi dire, l'auteur luimême qui opère dans ses personnages et le vouloir et l'opération. Il agit en tout agir de ses personnages. Pourtant, la décision du dramaturge que Tosca tue librement Scarpia n'implique pas que Tosca soit forcée par 
l'auteur dramatique de tuer Scarpia. On trouverait incongru que Meursault réponde au juge d'instruction que c'est Camus qui l'a poussé à tirer au revolver sur sa victime ${ }^{49}$.

Dans le cadre de l'analogie ici proposée entre, d'une part Dieu et le romancier ou dramaturge et, d'autres part les causes secondes et le scénario et les personnages du roman, le romancier comme tel n'appartient pas au récit. Si le romancier devait vraiment appartenir au récit, il devrait se décrire en train d'écrire qu'il écrit qu'il écrit etc... Un tel exercice devient rapidement impraticable ${ }^{50}$.

Nous avons deux opérations qui aboutissent au même résultat. Certes, nous ne connaissons pas de romancier ou dramaturge vivant et écrivant atemporellement. Au moins, c'est l'intérêt de cette analogie, la temporalité du scénario et des personnages est distincte de celle du romancier. Et s'il prend fantaisie au romancier de décrire son intervention, sa participation à la trame du roman, soit comme personnage fictif (point de vue de la première personne) soit réel (autobiographie), le temps de la composition de l'ouvrage ne se confond pas avec celui du déroulement de l'action. Et la distinction entre l'auteur (atemporel) et le narrateur (qui vous parle dans le temps du récit) subsiste. Lorsque Marcel Proust se met en scène comme narrateur dans la Recherche, il est d'un côté auteur et de l'autre

\footnotetext{
${ }^{49}$ Alain de Libera a écrit un roman où le héros demande à l'auteur de corriger le scénario, de refaire son caractère etc. Mais cette mise en abyme fait elle-même partie du roman : le personnage de Morgen Schtarbe (Flammarion, 1999) ne s'adresse pas plus à Libera que le héros de Rose Purple of Cairo (Woody Allen, 1985) ne s'échappe de l'écran pour conter fleurette à une spectatrice. C'est bien à l'écran qu'il sort de l'écran, c'est bien dans le roman que Morgen Schtarbe demande à changer le cours du roman.

${ }^{50}$ «La conception vraie (claironne un peu rapidement Maritain), la voici : le plan divin est immuable une fois fixé de toute éternité. Mais il n'est fixé de toute éternité que compte tenu de la libre défaillance de l'homme, que Dieu voit dans son éternel présent ». De cette manière «le plan divin ne doit pas être conçu anthropomorphiquement comme un scénario écrit d'avance » (Pour une philosophie de l'histoire, IV, Dieu et le mystère du monde, dans Oeuvres Complètes tome X 19521959, Editions Universitaires, Editions Saint Paul, Fribourg, Paris, 1985, p. 713). Maritain défend donc une fixité souple ! On laisse de côté l'idée que Dieu seul fait le bien, et que faire le mal c'est faire le rien (la fainéantise ou le "néantement"). Cette idée repose sur un jeu de mots. Maritain suggère que Sine me nihil potestis facere peut s'entendre "sans moi vous ne pouvez rien faire" aussi bien que "sans moi vous pouvez faire le rien" (p. 714). Ailleurs (Approche sans entraves, Fayard 1973, p. 315) Maritain s'en prend à «l'idée absurde et intolérable d'un Dieu Potentat-Dramaturge insensible dans son ciel au mal des personnages auxquels il fait jouer sa pièce de théâtre ». Mais il est possible, dans la conception atemporaliste de la création et de la conservation, d'affirmer que les personnages jouent librement, tout en tenant leur pouvoir d'agir et leur opération de Dieu seul, et que ce faisant, ils ne font pas rien, même quand ils font le mal. Et aussi, quand ils font le bien.
} 
personnage. Le point de vue de la première personne (du narrateur) fait alors partie du roman. Mais le romancier reste atemporellement responsable de l'existence et des agissements de ses personnages (il assume intégralement le scénario) et cependant, à l'intérieur de ce scénario, les produits de son imagination interagissent réellement selon la trame chronologique du roman ou de la pièce. Si c'est un bon romancier, il fera en sorte que ses personnages et le monde dans lequel ils évoluent en suivant un cours normal ou ordinaire (ce qui ne veut pas dire banal : mais les événements, les réactions, les décisions, les sentiments, même les plus inattendus, devront s'enchaîner selon des lois régulières). Un bon romancier ne se comportera pas, sauf exception, en deus ex machina. Non que cela lui soit impossible (il pourrait ainsi ressusciter chaque personnage décédé, altérer le fonctionnement des lois de la nature etc.), mais c'est une condition à respecter pour conférer une vraie responsabilité morale à certains personnages. Si le relèvement des cadavres est systématique, il n'y a plus vraiment d'homicide. S'il est chaotique, on ne sait plus à quoi s'en tenir. Le recours au deux ex machina doit donc rester exceptionnel (sauf dans un éventuel épilogue du roman).

Maritain a discuté cette métaphore de Dieu dramaturge, mais qui n'écrit pas de "scénario fait d'avance »: "Dieu a le cours tout entier du temps physiquement présent à son instant éternel, et qu'il l'a tout entier sous les yeux quand il fixe toutes choses de toute éternité [...] il n'y a pas de scénario fait d'avance, dont les agents créés tiendraient les rôles et seraient les exécutants [...] alors, poursuit Maritain, il faut bien que dans l'essentielle contingence du spectacle ainsi immuablement ordonné et dirigé par Dieu les personnages aient aussi leur part d'initiative, et même leur part d'initiative première dans le cas où il s'agit de l'initiative du mal, de l'initiative de cette défaillance ou de ce néantement dont la créature est cause première (déficiente) ${ }^{51}$.

Revenant sur la comparaison de la trame des événements avec un scénario d'écrivain, Maritain oppose "les auteurs de mauvais roman» (dont il nomme au passage quelques représentants contemporains selon lui) aux

\footnotetext{
${ }^{51}$ Dieu et la permission du mal, Paris- Bruges, Desclée de Brouwer, 1963, p. 86. Je remercie Antoine-Marie de Araujo d'avoir très précisément attiré mon attention sur ce texte.
} 
« vrais romanciers ». Les premiers sont déclarés coupables d'avoir écrit d'avance "sinon dans un temps antérieur au temps, du moins dans l'éternité supérieure au temps ", un "scénario où se trouve préfixé pour chaque personnage tout ce qu'il a à dire ou à faire sur le théâtre du monde ». Pour les seconds «ce sont leurs personnages en même temps qu'eux qui font le roman, ils résistent à l'auteur, ils n'en font qu'à leur tête, alors même que l'auteur suit le fil de son intuition créatrice avec une force souveraine $»^{52}$.

L'intuition de Maritain semble la suivante: dans un roman à thèse, les personnages se plient docilement aux volontés de l'auteur, sans consistance véritable. Le vrai romancier (Dostoïevski, Bernanos, Julien Green) laisse à ses personnages une véritable initiative. Pourtant, dans les deux cas, le caractère des personnages et l'intrigue sont tout autant intégralement dépendantes de l'auteur : il a écrit chaque ligne, pesé chaque mot. Si le «vrai romancier» donne l'illusion que ses personnages sont autonomes, c'est sans doute parce que, nous lecteurs, nous les trouvons ressemblants à nos congénères, ou à nous-mêmes. Ou que l'auteur s'est dit : « non Jean Valjean n'est pas un lâche : il va se dénoncer plutôt que de livrer un innocent». Mais cela reste une illusion, tout est écrit et fixé d'avance par l'auteur (et ses scrupules de vraisemblance). C'est lui, et lui seul qui fait exister Jean Valjean... Plutôt que d'essayer de perfectionner la comparaison (dont Maritain reconnaît le caractère défectueux : «la transcendance divine, à l'égard de laquelle toutes les notions tirées d'en bas ne sont que de très pauvres analogués $/^{53}$ ), il est loisible de recourir à la conception de la création atemporelle, qui évite l'idée de créatures se pliant docilement à un scénario écrit d'avance, non pas parce que Dieu ne serait plus l'auteur du scénario, mais parce qu'il ne l'écrit pas d'avance, et que son écriture atemporelle laisse la place à la contribution d'agents libres, qu'il connaît néanmoins atemporellement.

Enfin, pour achever cette évocation des contributions des philosophes analytiques contemporains au problème de l'incompatibilité entre

\footnotetext{
${ }^{52}$ Ibid. p. 88.

${ }^{53}$ Ibid., p. 89
} 
prescience et liberté, on pourrait évoquer la remarque de Nelson Pike, pionner de la redécouverte du problème de la temporalité divine : "this doctrine (of timelessness) is the ultimate expression of God's transcendence" ${ }^{\prime 4}$. On aurait alors un argument de convenance (semblable à celui qui attribue à Dieu la création ex nihilo, au sens où il convient mieux à un être tout-puissant d'opérer sans matériau préalable).

\footnotetext{
${ }^{54}$ Nelson Pike, God and Timelessness (Study in Ethics and Philosophy of Religion) Routlege and Kegan Paul 1970, Préface p. xi. Pike considère qu'il n'y a pas d'argument direct partant des autres attributs divins en faveur de l'atemporalité. Pike affirme que l'atemporalité implique l'immutabilité, l'incorruptibilité et l'immortalité, mais que la converse n'est pas vraie (1970: 43). On pourrait suggérer que l'atemporalité est la manière la plus simple d'être immuable, incorruptible et immortel. Un être atemporel n'a pas besoin de se prémunir contre le changement pour être immuable, d'échapper au vieillissement, de se maintenir en vie. Atemporel, il n'a même pas le temps de changer, de se corrompre, de mourir. Il est simplement immuable, incorruptible et immortel. On a essayé de montrer plus haut que ces attributs hiératiques n'enlevaient rien à sa connaissance du plus infime détail anthropologique et ne le laissaient pas dans l'ignorance de l'attente psychologique.
} 